\title{
Migrantes internos y mercado de trabajo en Tijuana, Baja California *
}

\author{
Lothar Witte**
}

El artículo presenta un ensayo de interpretación de la situación de ios migrantes en el mercado de trabajo de Tijuana. Se establece en primer término el marco teórico de referencia y se especifican las fuentes de datos utilizadas, entre ellas una encuesta aplicada a 672 unidades familiares de Tijuana en 1982. Para ubicar el análisis se hace referencia ai comportamiento demográfico de Tijuana y a la evolución de su mercado de trabajo. En ese contexto se lleva a cabo el análisis de la situación laboral de los migrantes mediante la comparación con la de la población nativa y en relación a sus antecedentes regionales y laborales. Se demuestra que los migrantes vienen de zonas de mayor desarrollo relativo; que la experiencia laboral se relaciona en forma directa con el carácter de desarrollo relativo de sus regiones de origen; que no existe diferencia con la población nativa en lo que se refiere a los sectores y posiciones en los que se insertan, y que el tipo de migración tiende a parecerse al que tiene lugar en los países industrializados más que a la que se da en los países en vías de desarrollo.

Una ciudad colmada de miles y miles de migrantes llegando a la puerta del paraíso, buscando su futuro. $Y$, muchas veces, destinados a sobrevivir en condiciones miserables, sin trabajo fijo y sin vivienda adecuada, en los cinturones de miseria. Éstos son los estereotipos sobre la vida de los migrantes en la ciudad fronteriza de Tijuana. Estereotipos que, sin duda tienen un fundamento sólido en un periodo de la historia de esta ciudad: entre 1920 y 1930 .

En las últimas décadas, no obstante, se han producido cambios profundos en Tijuana. Aunque ésta todavia tiene su zona roja y sigue siendo la puerta principal de los migrantes mexicanos a los Estados Unidos, se ha transformado en una ciudad donde también se encuentran industrias modernas, un sector terciario muy diversificado, instituciones académicas de buena reputación, etcétera.

Es de esperar que, con los cambios ocurridos, también haya cambiado el carácter del proceso migratorio. En este trabajo se presenta un ensa-

* En la elaboración de este trabajo, nos brindaron su apoyo una gran cantidad de instituciones e individuos. Una versión preliminar fue resultado de la colaboración entre la Universidad Libre de Berlin Occidental y el Centro de Estudios Fronterizos del Norte de México, hoy Colegio de la Frontera, en 1984; ésta contó con el apoyo financiero del Programa ASA de la Carl-Duisberg-Gesellschaft. Entre mis colaboradores, agradezco especialmente las contribuciones y los comentarios de Jorge Carrillo, Víctor Klagsbrunn, Corinna Kusel y Arturo Torres.

** Colaborador de la Fundación Friedrich Ebert, Bonn, República Federal Alemana. Las ideas contenidas en este trabajo son responsabilidad exclusiva del autor y no reflejan necesariamente las posiciones de los individuos o instituciones con las cuales está o estaba afiliado. 
yo de interpretación de un aspecto selecto de dicho proceso. Se trata de la situación de los migrantes en el mercado de trabajo de Tijuana, un mercado en continua transformación.

En el primer apartado se introducen brevemente los conceptos y las fuentes empleadas. En el segundo se presenta un resumen de la procedencia regional de los migrantes y de sus experiencias laborales en esas regiones. El tercero consta a su vez de tres subpartes. En la primera, se hace una breve referencia al desarrollo demográfico de Tijuana; en la segunda a los rasgos esenciales de la evolución del mercado de trabajo; en la tercera se lleva a cabo un análisis de la situación laboral de los migrantes, tanto en comparación con la población nativa como en relación a sus antecedentes regionales y sus experiencias laborales anteriores. Finalmente, en el último apartado se presenta una síntesis de los principales resultados.

\section{Conceptos}

Como cualquier otro fenómeno social de gran significado en la vida de las naciones, las migraciones internas son siempre históricamente condicionadas, siendo el resultado de un proceso global de cambio, del cual no deben ser separadas. Por tanto, el primer paso para su estudio es encontrar los límites de la configuración histórica que dan sentido a un determinado flujo migratorio (Singer, 1973:27).

Este tipo de consideraciones teóricas sobre el estudio de las migraciones internas puede entenderse como el núcleo programático de la escuela histórico-estructural: el proceso migratorio debe analizarse en el contexto del proceso de desarrollo.

Aunque esta perspectiva puso de relieve el paradigma de esta escuela, tanto contra los enfoques de la modernización sociocultural como de los trabajos de la economía neoclásica, resulta que frente a la producción de las ciencias sociales de épocas anteriores no representa una manera completamente nueva de proceder. Ya en el siglo pasado, Marx, tanto en sus principales obras teóricas como en sus trabajos sobre la emigración irlandesa, llamó la atención a las relaciones entre el proçeso de acumulación, el funcionamiento del mercado de trabajo y la movilidad de la fuerza de trabajo. ${ }^{1}$

Pero no solamente en el siglo pasado, sino después de la segunda guerra mundial se produjeron trabajos sobre migración que tuvieron en cuen-

${ }^{1}$ En El capital vale la pena considerar, por un lado, las elaboraciones sobre la llamada acumulación primitiva y, por otro, las referentes a la producción de la sobrepoblación relativa. También el capítulo sobre la gran industria tiene que ver con esta temática (Marx, 1979: 510512, 640 ss., 741 ss.). Los trabajos sobre la emigración irlandesa se encuentran en Marx (1969: 541-547), Marx (1971: 439-458), Marx (1979: 726 ss.). 
ta el contexto económico y social. La obra de Lewis (1954) constituye un ejemplo en este sentido.

Con los trabajos orientados a la metodología de la economía neoclásica, que pasaron a primer plano desde la década de los sesenta, cambió radicalmente el método (Todaro, 1969 y Harris y Todaro, 1970). En lugar del contexto económico y social, desde entonces el individuo maximizador de beneficios ocupa el centro de las consideraciones teóricas, un individuo abstracto que se concibe sin referencias al contexto. Sin embargo, también dentro de este paradigma se ha llamado la atención en los últimos años sobŕe la importancia de observar algo más que sólo un reducido número de factores económicos y sociales (Lipton, 1982; Sabot, 1982; Stark, 1982). Por el momento los enfoques orientados a la economía neoclásica no han logrado una metodología que verdaderamente ofrezca un análisis del proceso migratorio dentro del contexto económico y social. ${ }^{2}$

En contra de este enfoque todavía dominante, en lo que sigue se parte de que deben considerarse en primera línea los efectos de la estructura de un determinado mercado de trabajo sobre la situación laboral de los migrantes. Aunque los migrantes influyan en el mercado de trabajo al ofrecer una fuerza de trabajo con características socioeconómicas específicas, debe tenerse en cuenta en primera instancia el proceso de desarrollo particular de una región como condicionante del desarrollo del mercado de trabajo.

En segunda instancia, en un determinado mercado de trabajo las características socioeconómicas de los migrantes desempeñan un importante papel en la determinación de su situación laboral individual. En este trabajo se discuten principalmente las características que se forman durante la vida de los migrantes: las experiencias laborales y los niveles de calificación. Bajo este aspecto, la procedencia regional de los migrantes tiene un papel importante ya que, entre otros factores, las estructuras económicas y sociales de sus regiones de procedencia influyen considerablemente en la formación de las respectivas características socioeconómicas. En la medida en que estudiemos si la posición en el trabajo cambia o no antes y después del proceso de migración, podremos ofrecer además información relativa a una cuestión fundamental en el estudio de las migraciones: si contribuyen o no a la expansión del trabajo asalariado en una economía y sociedad en desarrollo. ${ }^{3}$

\footnotetext{
${ }^{2}$ En este trabajo, sólo es posible delinear unos rasgos muy generales de los enfoques neoclásicos. Una evaluación crítica más profunda se encuentra en Witte (1986a: 14-33).

${ }^{3}$ Esta contribución está entre las consideraciones principales, por ejemplo, de Lewis (1954) y de Singer (1973). Este último escribe: "Las migraciones internas desempeñan un papel de gran importancia en estas transformaciones de las estructuras económicas y sociales. El paso de parte de la población de una clase a otra, muchas veces se produce mediante movimientos en el espacio", Singer (1973:49).
} 
En este trabajo, además de la literatura disponible y de las impresiones obtenidas durante una estancia prolongada en Tijuana en 1984, pude aprovechar algunas fuentes inéditas. En primer lugar, utilizamos datos levantados por el Centro de Estudios Fronterizos del Norte de México (Cefnomex) -ahora llamado Colegio de la Frontera (Colef)- en abril de 1982. El relevamiento de datos incluyó seis ciudades fronterizas; en Tijuana fueron encuestadas 672 unidades familiares. ${ }^{4}$ En segundo lugar, aprovechamos datos de una investigación llevada a cabo por el Instituto de Investigaciones Sociales de la Universidad Autónoma de Baja California (iiS-UABC), en junio de 1984, en la cual se investigaron 4501 personas en 831 hogares. Además, se nos facilitaron datos inéditos sobre el mercado de trabajo. ${ }^{5}$

Los conceptos utilizados en este trabajo están fuertemente condicionados por las fuentes disponibles. En lo que se refiere a la categoría de migrante, se trata de la migración habitualmente llamada "permanente", la migración que lleva a una instalación de la residencia habitual en el destino de la migración. En la elaboración de las respectivas categorías en el mercado de trabajo, se sigue el principio de garantizar una cierta comparabilidad con otras fuentes, sobre todo con los censos. En el apéndice se elaboran con más detalle estas categorías.

\section{Procedencia regional y experiencias laborales anteriores}

La procedencia regional de los migrantes y sus características socioeconómicas, en parte vinculadas a la procedencia regional, cuentan entre las principales determinantes de los aspectos cualitativos de su fuerza de trabajo. Al ofrecer ésta en el mercado de trabajo de destino, los migrantes contribuyen también al desarrollo específico de dicho mercado y de la región respectiva. En lo que sigue, se presenta por un lado un resumen de la procedencia regional de los migrantes hacia la ciudad de Tijuana, y por otro lado, los rasgos principales de sus experiencias laborales en esas regiones de procedencia.

${ }^{4}$ Este estudio tuvo inicialmente el carácter de una encuesta de opinión política, pero ya ha sido tomado en cuenta en trabajos sobre la migración. Véanse Bustamante (1982) y (1984). Los datos no procesados fueron nuevamente evaluados por el autor durante una estancia en el Cefnomex en 1984. En el apéndice se describe en más detalle este proyecto de Carrillo y Witte (1984). En el trabajo aquí presentado, siempre que no se señale lo contrario, los datos provienen de este proyecto.

${ }^{5}$ IIS-UABC (1975). Entre tanto, el reporte de investigación ha sido revisado. En la medida en que en este trabajo no me sirvo de las interpretaciones sino principalmente de los datos levantados por el IIS-UABC, parece justificado no haber consultado la versión final. En esta ocasión, quiero agradecer a los colaboradores de este instituto por la ayuda que me brindaron. 


\section{Procedencia regional}

El cuadro 1 muestra que los migrantes provienen en gran parte de las regiones en las cuales se encuentra la zona fronteriza (noroeste, norte, golfo) pero de manera considerable también de estados meridionales (centrooeste, ciudad de México). ${ }^{6}$ Mientras que Ciudad Juárez y Nuevo Laredo asumen en gran medida la función de centros regionales, las ciudades bajacalifornianas de Mexicali y especialmente Tijuana atraen altos porcentajes de migrantes del centro-oeste y de la zona metropolitana de la ciudad de México. En lo que se refiere al nivel de desarrollo de esas regiones, especialmente las norteñas y la capital están fuertemente caracterizadas por una producción para el mercado, el trabajo asalariado, un nivel relativamente desarrollado de las fuerzas productivas, así como por una alta participación del sector secundario y terciario en el producto regional y en la población económicamente activa. Pero también en el centro-oeste se han producido en las últimas décadas considerables procesos de transformación. En consecuencia, tanto en lo que respecta al grado de urbanización como a los indicadores económicos esta región adquiere una posición intermedia entre regiones desarrolladas y menos desarrolladas (véase Unikel, 1978: 177-212 y Hernández Laos, 1984). En resumen, se pone en evidencia la gran importancia que la migración desde las regiones desarrolladas del país tiene para la región fronteriza del norte.

¿Cuáles podrían ser las causas subyacentes de esa estructura de procedencia? Limitando la discusión al caso más interesante (la ciudad de Tijuana), proponemos discutir dos elementos principales: por un lado, las tradiciones migratorias formadas en el curso del desarrollo histórico, y por otro, el cambio general de los movimientos demográficos mexicanos observado desde la década de los setenta.

En lo que respecta a las tradiciones migratorias, es importante destacar que Tijuana se encuentra en el radio de atracción de importantes corrientes migratorias temporarias hacia los Estados Unidos y también hacia regiones de agricultura capitalista del noroeste de México. Aunque el origen reciente de los "golondrinos" se encuentra al sur del país, durante décadas estas corrientes fueron alimentadas principalmente por migrantes provenientes del centro-oeste y de estados vecinos. ${ }^{7} \mathrm{Al}$ mismo tiem-

${ }^{6}$ En la regionalización del territorio mexicano se sigue la división propuesta por Unikel (1978): noroeste (Baja California Norte, Baja California Sur, Nayarit, Sinaloa, Sonora), norte (Coahuila, Chihuahua, Durango, Nuevo León), golfo (Tamaulipas, Veracruz), centro-norte (Aguascalientes, San Luis Potosí, Zacatecas), centro-oeste (Colima, Guanajuato, Jalisco, Michoacán), centro (Hidalgo, Morelos, Puebla, Querétaro, Tlaxcala), zona metropolitana de la ciudad de México (Distrito Federal, estado de México), sur (Campeche, Chiapas, Guerrero, Oaxaca, Quintana Roo, Tabasco, Yucatán).

7 Por ejemplo, según Zazueta (1980: 86), 40.2\% de los migrantes mexicanos en los Estados Unidos provienen sólo de los tres estados: Guanajuato, Jalisco y Michoacán. 
CUADRO 1

Regiones de procedencia de los migrantes entrevistados en 1982 en ciudades seleccionadas de la región fronteriza del norte de México

\begin{tabular}{lrrcr}
\hline \multicolumn{1}{c}{$\begin{array}{c}\text { Región de } \\
\text { Procedencia }\end{array}$} & Tijuana & Mexicali & Ciudad Juárez & Nuevo Laredo \\
\hline Noreste & 37.2 & 49.1 & 3.7 & 1.5 \\
Norte & 10.6 & 10.4 & 75.5 & 38.4 \\
Golfo & 0.6 & 0.6 & - & 16.7 \\
Centro-norte & 3.1 & 2.3 & 10.8 & 15.3 \\
Centro-oeste & 30.5 & 27.7 & 5.8 & 15.8 \\
Centro & 1.6 & - & 1.2 & 1.0 \\
Zona metropolitana de & & & & \\
$\quad$ la ciudad de México & 14.3 & 7.5 & 2.5 & 7.9 \\
Sur & 1.8 & 2.3 & 0.4 & 3.4 \\
Total & 100.0 & 100.0 & 100.0 & 100.0 \\
& $(511)$ & $(173)$ & $(241)$ & $(203)$ \\
\hline
\end{tabular}

Fuente: Jorge Carrillo y Lothar Witte, "Migración y transformación social: migrantes mexicanos en la frontera norte de México y en los Estados Unidos", Tijuana, 1984, materiales no publicados.

po, el noroeste mismo pasa a ser la región en que el incremento de la productividad en la agricultura se vincula con movimientos de emigración orientados esencialmente a las ciudades del noroeste y sólo de una manera muy reducida hacia otras regiones (Unikel, 1978: 213-245, Stern, 1977). Con base en estas corrientes migratorias establecidas en el curso del tiempo, y en el crecimiento de Tijuana, se han establecido relaciones económicas y sociales entre los que ahora viven en esas ciudades y los familiares o amigos que permanecen en su región que facilitan la migración. Estas redes sociales permiten entender que un movimiento migratorio procedente de una localidad en Michoacán o Jalisco hacia la lejana Tijuana, sólo representa un paso pequeño en términos de distancia social. Entonces, los migrantes no llegan aislados y "buscando su futuro" sino muchas veces preparados para integrarse a una microestructura social que les facilita la reproducción. Como esa microestructura social comprende tanto la región de procedencia como la de destino, se puede decir que de alguna manera las tradiciones migratorias reproducen continuamente la estructura de procedencia de los migrantes (véase Henning/Paulsdorff, 1985 y Witte, 1986b). Pero no solamente los caminos conocidos determinan las corrientes migratorias: éstas reflejan la transformación de la dinámica de los movimientos demográficos observada en la última década. Aunque la población de Tijuana también se vio afectada por el deterioro de las condiciones de vida 
después de 1976, hasta 1982 se destacó en relación con otras ciudades por sus condiciones de vida relativamente privilegiadas. Por ende, la ciudad atrajo migrantes también de otras regiones urbanas e incluso de las grandes ciudades del país. Por ejemplo, los datos del censo de 1980 sugieren que la migración de la ciudad de México ha cobrado importancia en la década de 1970. En 1970 los migrantes de la capital representaban 9.3\% del total de migrantes provenientes de otros estados. En 1980, entre los migrantes que habían vivido no más de cuatro años en Tijuana, ya eran $15.4 \%{ }^{8}$ Según los datos que dieron origen al cuadro 1 , si se suman los que antes de la migración vivían en Guadalajara a los que vinieron de la ciudad de México, resulta que más de la cuarta parte del total vivía antes en una de las dos grandes metrópolis. Considerando además el nivel de urbanización generalmente elevado del noroeste y del centro-oeste, ha de admitirse que la migración desde regiones urbanas es muy significativa para Tijuana.

Resumiendo, en el caso de la migración a Tijuana se entrelazan las corrientes establecidas de las regiones del noroeste y centro-oeste con los movimientos desde las grandes metrópolis del país para dar como resultado el predominio de las regiones relativamente desarrolladas entre las principales regiones de procedencia de los migrantes.

\section{Experiencias laborales anteriores}

El que los migrantes hacia la región fronteriza y particularmente a Tijuana vengan en su mayoría de las regiones más desarrolladas del país no quiere decir que tengan en su región de procedencia un trabajo asalariado. Es verdad que, teniendo en cuenta la amplitud del trabajo asalariado en las principales regiones de procedencia, se puede suponer que los migrantes tenían en gran parte una ocupación asalariada antes de trasladarse a Tijuana. No obstante, también es posible que los migrantes se reclutaran precisamente del grupo de los productores antes independientes que no tuvieron ninguna posibilidad de seguir sobreviviendo de este modo en su región de origen.

Según los datos presentados en el cuadro 2, la mayor parte de los migrantes hacia la región fronteriza que también habian ejercido en su origen una actividad económica, tuvieron allí una ocupación asalariada. Alrededor de $40 \%$ se desempeñaron como empleados, más de $10 \%$ como obreros sólo eñ industrias de transformación y en la construcción, y casi $13 \%$ como jornaleros agríeolas. Este cuadro también muestra las diferencias según las regiones de procedencia: los migrantes provenientes de regiones en las que se han impuesto con mayor intensidad las relaciones 
capitalistas tenían ocupaciones asalariadas en un porcentaje que supera al promedio. De este modo, entre los migrantes de la ciudad de México, sustancialmente más de la mitad eran empleados y $12 \%$ desempeñaban profesiones liberales y técnicas. También en el noroeste los migrantes eran con frecuencia empleados ( $47 \%$ ), mientras que la intensa penetración capitalista en la agricultura de esa región se refleja en el elevado porcentaje de jornaleros dedicados a la agricultura ( $12 \%$ sobre un total de $14.7 \%$ del sector agrícola). En el centro-oeste, a pesar de que también allí los empleados ocupan casi una tercera parte, el porcentaje de migrantes que proviene del sector agrícola es elevado (más de $30 \%$ ), algo que refleja por un lado la gran importancia del empleo agrícola en esa región y por otro

\section{CUADRO 2}

Experiencias laborales anteriores de lo migrantes internos entrevistados en 1982 en la región fronteriza del norte de México*

\begin{tabular}{lrrrrr}
\hline & \multicolumn{5}{c}{ Región de procedencia } \\
\cline { 2 - 6 } Posición en el trabajo en & \multicolumn{6}{c}{} & $\begin{array}{c}\text { Ciudad } \\
\text { de } \\
\text { la región de procedencia }\end{array}$ & Total * & Noroeste & Norte & Centro-oeste & México \\
\hline Profesionistas y técnicos & 4.7 & 4.4 & 2.3 & 2.5 & 12.0 \\
Empleados & 40.3 & 47.0 & 43.1 & 31.9 & 57.3 \\
$\quad$ Comercio & 14.2 & 14.3 & 13.6 & 12.9 & 21.3 \\
$\quad$ Servicios & 15.0 & 20.7 & 10.2 & 10.4 & 22.7 \\
Obreros y artesanos & 16.0 & 15.5 & 19.9 & 17.2 & 8.0 \\
$\quad$ Ind. de transformación & 5.2 & 3.6 & 9.7 & 3.1 & 5.3 \\
$\quad$ Construcción & 5.1 & 5.2 & 4.5 & 7.4 & 1.3 \\
Ocupados en agricultura & 20.1 & 14.7 & 22.7 & 31.1 & 2.7 \\
$\quad$ Jornaleros & 12.9 & 12.0 & 10.8 & 20.9 & 2.7 \\
Independientes & 18.9 & 18.3 & 21.0 & 17.2 & 20.0 \\
$\quad$ Comercio & 2.6 & 4.4 & - & 3.7 & 1.3 \\
$\quad$ Servicios & 10.7 & 7.6 & 16.5 & 8.6 & 9.3 \\
Total & 100.0 & 100.0 & 100.0 & 100.0 & 100.0 \\
& $(745)$ & $(251)$ & $(176)$ & $(163)$ & $(75)$ \\
\hline
\end{tabular}

* Aquí están consideradas las ciudades de Tijuana, Mexicali, Ensenada, Tecate, Ciudad Juárez, Nuevo Laredo.

** Están incluidos los migrantes de regiones no indicadas por separado.

Fuente: Jorge Carrillo y Lothar Witte, "Migración y transformación social: migrantes mexicanos en la frontera norte de México y en los Estados Unidos", Tijuana, 1984, materiales no publicados. 
los importantes procesos de transformación que sufre dicho sector. Entre los migrantes alli ocu pados en la agricultura, de nuevo destaca el elevado porcentaje de jornaleros, aunque también relativamente muchos productores, hasta el momento de la migración independientes, se vieron obligados a emigrar. Resumiendo, la migración hacia la frontera norte no es de pequeños propietarios agrícolas o de trabajadores independientes del sector terciario, sino principalmente se trata de un movimiento de asalariados.

En el cuadro 3 se puede observar que existen también diferencias relevantes entre las experiencias laborales anteriores de los migrantes si se considera su lugar de destino. Por ejemplo, entre los migrantes que llegaron a Tijuana y Nuevo Laredo, el porcentaje que antes de la migración trabajaba como empleados, obreros/artesanos o jornaleros resulta ser más de $\mathbf{7 0} \%$, mientras que las cifras correspondientes para Mexicali y Ciudad Juárez son poco más de 60 y $55 \%$, respectivamente. Por un lado, estos resultados reflejan otra vez las diferencias en la estructura económica de las regiones de procedencia de los migrantes. Por ejemplo, el porcentaje relativamente alto de trabajadores industriales entre los migrantes a Nuevo Laredo, entre los cuales $12 \%$ trabajaba antes en industrias de transformación, refleja el alto nivel de industrialización del noreste mexicano. Por otro lado, las diferencias entre las experiencias laborales de los migrantes a Tijuana y Mexicali no se pueden explicar sólo por diferencias en el origen regional. Dado que la estructura de procedencia es muy parecida para ambas ciudades, se supone que la estructura económica del lugar de destino también ejerce una influencia selectiva sobre la migración. Es así como los campesinos y jornaleros raramente encontrarán en Tijuana un "campo natural " y en el marco de la migración laboral temporaria entrarán en contacto menos con Tijuana que con los municipios vecinos de Mexicali y Ensenada. En consecuencia, también la migración permanente aquí estudiada en el caso de Mexicali está intensamente marcada por migrantes del campo. Por el contrario, los migrantes llegados a Tijuana frecuentemente estaban ocupados antes de la migración como empleados en el sector terciario (34.2\%), mientras que el trabajo agrícola era de menor significación.

Los resultados que destacan son, en primer lugar, que los migrantes hacia la región fronteriza del norte y especialmente a Tijuana provienen en su mayor parte de las regiones más desarrolladas del país, donde el nivel de urbanización es alto y las relaciones capitalistas dominan la economía. En segundo lugar, de acuerdo con esta estructura socioeconómica de sus regiones de procedencia, las experiencias laborales de los migrantes destacan por el predominio del trabajo asalariado, sobre todo en la posición de empleados. En tercer lugar, las experiencias laborales difieren de acuerdo con las estructuras socioeconómicas particulares de las respectivas regiones de procedencia como también de destino: entre más desarrollada la región, más destaca el predominio del trabajo asalariado entre las experiencias laborales de los migrantes. 


\section{CUADRO 3}

Experiencias laborales anteriores de los migrantes internos entrevistados en 1982 en ciudades seleccionadas de la región fronteriza del norte de México

\begin{tabular}{lrrrr}
\hline $\begin{array}{l}\text { Posición en el trabajo en } \\
\text { la región de procedencia }\end{array}$ & Tijuana & Mexicali & Ciudad Juárez & Nuevo Laredo \\
\hline Profesionistas y técnicos & 5.4 & 5.3 & 2.2 & 4.0 \\
Empleados & 45.4 & 27.6 & 36.0 & 39.4 \\
$\quad$ Comercio & 16.5 & 6.6 & 14.6 & 13.1 \\
$\quad$ Servicios & 17.7 & 15.8 & 11.2 & 12.1 \\
Obreros y artesanos & 13.8 & 13.2 & 9.0 & 21.2 \\
$\quad$ Ind. de transformación & 5.0 & 2.6 & 2.2 & 12.1 \\
Construcción & 5.0 & 3.9 & 2.2 & 5.1 \\
Ocupados en agricultura & 17.7 & 31.6 & 25.8 & 25.3 \\
$\quad$ Jornaleros & 13.1 & 21.1 & 10.1 & 12.1 \\
Independientes & 17.7 & 22.4 & 27.0 & 10.1 \\
Comercio & 3.1 & 3.9 & 1.1 & - \\
Servicios & 8.8 & 10.5 & 21.3 & 7.1 \\
Total & 100.0 & 100.0 & 100.0 & 100.0 \\
& $(260)$ & $(76)$ & $(89)$ & $(99)$ \\
\hline
\end{tabular}

Fuente: Jorge Carrillo y Lothar Witte, "Migración y transformación social: migrantes mexicanos en la frontera norte de México y en los Estados Unidos", Tijuana, 1984, materiales no publicados.

\section{Migrantes internos y mercado de trabajo en Tijuana}

En este apartado se presentan las interrelaciones entre el desarrollo urbano de Tijuana, la estructura y el funcionamiento del mercado de trabajo, y la situación laboral de los migrantes. Para empezar, se presenta un resumen del desarrollo demográfico de la ciudad. En segundo lugar, se elaboran los rasgos esenciales de la evolución del mercado de trabajo desde la década de los cincuenta, llegando a una presentación de la estructura del mercado de trabajo en los años setenta y ochenta. Con base en este contexto general, se presenta el análisis de la situación de los migrantes: por un lado, en comparación con los nativos y, por otro, según sus experiencias laborales anteriores y su procedencia regional.

Crecimiento poblacional y migración interna en Tijuana

La ciudad de Tijuana, en la que hasta principios de este siglo vivían apenas unas 250 personas, experimentó su gran crecimiento durante el periodo posterior a la segunda guerra mundial. En 1940 vivían allí alrede- 
dor de 22 mil personas. En las décadas siguientes y hasta 1970, el ritmo de expansión llegó al punto de que era habitual una duplicación de la población en menos de 10 años, de tal manera que en 1970 la población residente ascendió a 340 mil personas (SIC; 1976). Se supone que en la década de los setenta, la población se expandió a un ritmo todavía mayor que en las otras metrópolis del país, y se estima que para principios de los ochenta la población era de entre 700 y 800 mil habitantes. ${ }^{9}$

Este crecimiento demográfico se debe en gran medida a la migración interna. En los setenta y ochenta, alrededor de la mitad de la población residente son migrantes de otros estados mexicanos. ${ }^{10} \mathrm{Si}$ se incluyen además los migrantes de otras áreas de Baja California Norte, es de suponer que casi dos de cada tres residentes de Tijuana no habían nacido en esta ciudad. ${ }^{11}$ En vista de que gran parte de los migrantes vinieron a Tijuana en edad reproductiva, resulta que también una parte esencial del crecimiento natural representa un aporte indirecto de la migración al crecimiento demográfico. En resumen, no cabe duda de que en las últimas décadas la ciudad de Tijuana ha representado el prototipo de una ciudad que crece por la migración.

Corriendo el peligro de una simplificación excesiva, podríamos decir que los principales factores que atrajeron a estos migrantes fueron el desarrollo de la economía urbana y, correspondientemente, la expansión del mercado de trabajo; y, en segundo lugar, las condiciones de vida relativamente privilegiadas.

En lo que respecta al desarrollo de la economía urbana, hasta los años cincuenta y sesenta estaba orientada hacia dos rubros principales. Por un lado, la oferta de servicios para ciudadanos norteamericanos que venían a Tijuana para disfrutar de servicios no ofrecidos o más caros en su país, y por otro, la oferta de servicios para ciudadanos mexicanos que venían a Tijuana para trasladarse al otro lado de la frontera. Aunque ambas áreas todavía tienen importancia en la economía de Tijuana, con el desarrollo

${ }^{9}$ El Censo de Población de 1980 sólo indica un número de 461257 habitantes; spP (1983a). No obstante, los datos no fueron adecuadamente levantados, lo que llevó a una subestimación sustancial de la población residente (Corona, 1983). En cambio, las estimaciones disponibles antes de la publicación de los datos censales por lo general partian de que en 1980 el número de habitantes estaría entre 600000 y 750 000; Guevara Santillán (1979:11), espera una cifra de 741867 habitantes. Melesio Nolasco (1983:102), llega al número de 728514 y Nafinsa (1984:28), a 624000 habitantes.

${ }^{10}$ Sic (1976), sPP (1983a); cálculos propios.

11 Según los datos de Carrillo y Witte (1984) e us-UABC (1985) se llega incluso al resultado de que los migrantes representan más del $75 \%$ de la población. No obstante, a causa de la metodología empleada, en estos estudios la proporción de los migrantes está sobrestimada. En el proyecto de Carrillo y Witte (1984), la elevada proporción resulta de la elección de los jefes de familia como unidades de investigación, debido a la cual los hijos nacidos en Tijuana, quienes reducirían el porcentaje de migrantes en la población total, no fueron tenidos en cuenta. La investigación del IIS-UABC (1985), se llevó a cabo principalmente en colonias relativamente nuevas en las que los migrantes están sobrerrepresentados. 
de la ciudad la economía interna creció y se diversificó. En las décadas de los sesenta y setenta este desarrollo fue influido por una serie de programas estatales (Programa Nacional Fronterizo, Programa de Industrialización Fronteriza, Programa de Comercialización Fronteriza, etc.). Aunque es dudoso en qué medida estos programas lograron sus objetivos, contribuyeron a la expansión y diversificación del mercado de trabajo.

En lo que se refiere a las condiciones de vida prevalecientes en estos años, existen dos opiniones opuestas. Por un lado, circularon verdaderas leyendas sobre la buena vida en la frontera; por el otro, hay descripciones de Tijuana que la declaran el basurero del país. Aunque es difícil saber cuál de las dos opiniones es correcta, considerando el conjunto de la evidencia disponible es probable que, en promedio, en la época de la posguerra y hasta 1982, las condiciones de vida fueron mejores que en el interior del país. A pesar de que el costo de la vida era superior a la del interior, los salarios más altos del país y las posibilidades de trabajar temporalmente en los Estados Unidos bastaban para lograr un nivel de vida relativamente privilegiado. ${ }^{12}$

\section{Evolución del mercado de trabajo}

La historia del desarrollo de Tijuana como centro comercial y de servicios también se refleja en la estructura del empleo. En lo que respecta a las ramas de actividad, el sector terciario fue siempre el más importante después de la segunda guerra mundial. Ya en 1950 más de la mitad de la población económicamente activa se concentraba en este rubro, y se estima que para los principios de los ochenta aproximadamente dos de cada tres ocupados trabajaban en el terciario. La ocupación en la industria se expandió también en términos absolutos y relativos, aunque de manera menos espectacular que en el sector terciario. El sector agrario, en cambio, perdió significación. En 1950, el sector todavía empleaba alrededor de un cuarto de todos los ocupados, mientras que hoy en día son pocas las personas que se encuentran trabajando en el campo. ${ }^{13}$ En estos datos se refleja, por

${ }^{12}$ En este trabajo no es posible elaborar más estos aspectos. Véanse, por ejemplo, las contribuciones compiladas por Klagsbrunn (1986a) Mungaray y Moctezuma (1985), Price (1973) y Witte [1986], para elaboraciones más completas.

${ }^{13}$ Para 1950-1970, Conapo (1984) y cálculos propios. Para 1980, según cálculos propios con base en el censo (SPP, 1983a), resulta lo siguiente: $16.5 \%$ comercio, $3.9 \%$ transporte, $18.9 \%$ servicios, $24 \%$ no especificados. Conforme a cálculos propios con base en el estudio realizado por el IIS-UABC (1985), en 1984, 17.5\% se ocupa en el comercio, $3.9 \%$ en el transporte y $4.7 \%$ en el sector de servicios. Es probable entonces que la mayoría de los ocupados que según el censo no pudieron ser clasificados, trabajen en el sector servicios de Tijuana. En comparación con los resultados del censo de 1970 , según el cual $57.6 \%$ trabajaba en el sector terciario, y ante el desarrollo de la economía urbana en la década de los setenta, los datos indicados aparecen justificados, o por lo menos plausibles. Para argumentos parecidos véase también Mungaray y Moctezuma (1985). 
un lado, la transformación del trabajo de los migrantes en los Estados Unidos. En las décadas de los cincuenta y los sesenta, tal trabajo era principalmente en el sector agrario. En los ochenta, más de $40 \%$ de los transmigrantes provenientes de Tijuana en los Estados Unidos trabajan en el sector servicios (IIS-UABC, 1984). Por otro lado, esta evolución descansa en el desarrollo dinámico de los sectores secundario y terciario de la ciudad, como también en el deterioro general de las condiciones de vida en las décadas de los setenta y ochenta. De esta manera, la población en edad de trabajar tanto tiene las posibilidades como se ve obligada a conseguir una actividad pagada. Correlativamente, en la década de los setenta la población económicamente activa aumentó a un ritmo considerablemente mayor que la población en edad de trabajar (Mungaray y Moctezuma, 1985:43).

En contra de la difundida opinión de que una economía urbana dominada por el sector terciario tendría que estar también caracterizada por el trabajo independiente, en Tijuana predomina el trabajo asalariado. Ya en $1970,70 \%$ de la población económicamente activa era asalariada. ${ }^{14}$ Según investigaciones empíricas realizadas en los primeros años de los ochenta, durante esa época el porcentaje de los asalariados también era de alrededor de $70 \% \cdot{ }^{15}$ Como era de esperar, el trabajo asalariado predominaba en grado diferente según la rama de actividad. Mientras que en la industria y en el sector servicios más de $70 \%$ de los ocupados trabajaban en posiciones asalariadas, en el comercio constituían apenas $60 \%{ }^{16}$ No obstante, estos números bastan para relativizar otra vez la supuesta importancia del trabajo independiente en "economías terciarizadas".

Con respecto al trabajo asalariado, ha de considerarse, sin embargo, que su elevada proporción no debe equipararse apresuradamente con un predominio del trabajo asalariado bajo condiciones capitalistas. Todavía en los primeros años de los setenta era característico tanto del sector secundario como del terciario que a un reducido número de grandes empresas se contraponía una enorme cantidad de pequeños establecimientos. Así, para 1973 un estudio de más de 10000 establecimientos urbanos mostró que menos de $1.5 \%$ de las empresas concentraban más de $60 \%$ del capital invertido, mientras que a $84 \%$ de las mismas correspondió menos de $15 \%$ del capital. ${ }^{17}$ No obstante, desde la década de los setenta se impusieron relaciones capitalistas con mayor intensidad. En el sector secundario, este desarrollo se debió principalmente al crecimiento de la indus-

${ }^{14}$ SIC (1976); cálculos propios.

${ }^{15}$ Según los datos de Carrillo y Witte (1984), para 1982 se llega a $54.1 \%$ de empleados y $11.7 \%$ de obreros y artesanos. Según cálculos propios con base en los datos del IIS-UABCC (1985), en 1984 los porcentajes fueron $58.9 \%$ de empleados y $14.8 \%$ de obreros. Mungaray y Moctezuma (1985), indican para $1984,40.2 \%$ y $21.7 \%$, respectivamente. Si se incluyen a los ocupados como técnicos, que a menudo son asalariados, resulta el porcentaje indicado.

${ }^{16}$ SPP (1983a); cálculos propios.

${ }^{17}$ Guevara Santillán (1979: 150 ss.); cálculos propios. 
tria maquiladora. Mientras que en los últimos años de los setenta el empleo en este sector permaneció bajo el marco de diez mil, a principios de 1984 ya más de veinte mil personas trabajan allí (SPP, 1985b y 1984). Así, en los primeros años de los ochenta la mayor parte de los ocupados en la industria de transformación trabajaba en maquiladoras. ${ }^{18}$ En el sector terciario, por un lado, desempeñaron un papel importante las medidas estatales para la constitución de un sector moderno. Por otro lado, la forzada orientación del consumo de la población hacia productos nacionales llevó a que desde 1982 el capital nacional se comprometiera más en Tijuana para aprovechar las ventajas competitivas de que goza, gracias a la devaluación, frente a empresas que operan en los Estados Unidos. Algunas grandes empresas comerciales mexicanas, nunca antes representadas en $\mathrm{Ti}$ juana, compraron empresas locales menores, mientras que las firmas más importantes, ya establecidas antes de 1982, extendieron desde entonces sus actividades (Mungaray y Moctezuma, 1985: 189 ss. y 1984). De este modo, se han producido considerables procesos de centralización que condujeron a una creciente penetración capitalista del sector terciario. A pesar de que no se dispone de datos particulares, con base en la transformación general de la economía urbana parece evidente que también el trabajo asalariado bajo condiciones capitalistas se expandió en estos últimos años.

Aunque no existen estudios empíricos sobre esa temática, puede suponerse con base en la información disponible que la evolución descrita de la economía urbana se expresa, mediante las prácticas de reclutamiento de las empresas y de las instituciones públicas, en una absorción de mano de obra selectiva: mientras que la población joven y bien calificada puede aprovechar las oportunidades de un mercado de trabajo en expansión, la creciente disponibilidad de ese grupo permite a los empresarios prescindir de los más viejos y menos calificados. Es muy significativo al respecto la evolución de la estructura demográfica. En las últimas décadas, se ha incrementado constantemente el potencial de mano de obra disponible; las personas mayores de 12 años representan en Tijuana un creciente porcentaje en el total de la población. Dentro de esta población en edad de trabajar aumenta constantemente también la proporción de los individuos de entre 15 y 29 años. En 1980, casi la mitad de la población económicamente activa pertenece a este grupo (SPP, 1983a y Mungaray y Moctezuma, 1985: 37ss.). En lo que respecta a su posición en el trabajo, este grupo trabaja en posiciones asalariadas en una proporción significativamente mayor al promedio. Según un estudio realizado en $1984,80 \%$ de los ocupados de esta edad eran asalariados. ${ }^{19}$ En cambio, a los candidatos de más edad a menudo les está vedado el acceso a los sectores mo-

\footnotetext{
${ }^{18}$ Carrillo (1984), IIS-UABC (1985), cálculos propios.

19 IIS-UABC (1985); cálculos propios.
} 
dernos de la economía; se ven crecientemente obligados o a emprender una actividad independiente o a retirarse por completo de la actividad económica, sobre todo cuando por lo demás sólo cuentan con una escasa calificación formal. En Tijuana, esta calificación en el curso del desarrollo de la economía urbana se ha ido convirtiendo en un requisito obligatorio para determinadas posiciones en el mercado de trąbajo. No sólo la ocupación como profesionistas y técnicos, sino también la actividad asalariada en el sector terciario o en las maquiladoras exige a menudo la aprobación de la secundaria. Este requisito es actualmente satisfecho por más de la mitad de las personas entre 15 y 29 años. ${ }^{20}$ En estas circunstancias parece lícito concluir que en Tijuana se trata de una fuerza de trabajo en cantidad y calidad adecuada para el trabajo asalariado en condiciones capitalistas.

Resumiendo, para los primeroś años de los ochenta, el mercado de trabajo en Tijuana estaba dominado por el sector terciario, en el cual trabajaban dos de cada tres ocupados. En segundo lugar estaba la industria, mientras que la agricultura no era significativa. Entre las posiciones en el trabajo, en todas las ramas predominaba el trabajo asalariado con alrededor de 70\%. En la industria, la gran mayoría de los asalariados trabajaba bajo condiciones capitalistas. En el sector terciario, se observaba por lo menos una creciente penetración capitalista. Existía una gran disponibilidad de mano de obra joven y calificada, lo que mediante las prácticas de reclutamiento resultaba en una absorción selectiva de la fuerza de trabajo según la cual este grupo trabajaba en posiciones asalariadas en proporciones todavía mayores al promedio.

Migrantes internos en el mercado de trabajo de Tijuana

Migrantes y nativos en el mercado de trabajo

En varios estudios empíricos realizados en diversas grandes ciudades mexicanas, se manifestó que la situación laboral de los migrantes no difiere en gran medida de la de la población nativa. ${ }^{21}$ En el caso de la ciudad de Tijuana, que se expandió en las últimas décadas de una manera todavía más rápida que las otras ciudades mexicanas, ¿no tendría uno que esperar resultados diferentes?

Según los datos presentados en el cuadro 4 , también en Tijuana la situación laboral de los migrantes (los cuales representan casi $75 \%$ de las personas ocupadas), corresponde en gran medida a la de la población na-

\footnotetext{
${ }^{20}$ Según el estudio del IIS-UABC (1985), hasta $60.7 \%$ de las personas de entre 20 y 25 años y $51.4 \%$ de aquellas entre 25 y 29 años han completado la secundaria; cálculos propios.

${ }^{21}$ Véanse Balan, Browning y Jelin (1973) para el caso de Monterrey, y Muñoz, Oliveira y Stern (1977), para el Distrito Federal.
} 
tiva. En ambos grupos, alrededor de dos de cada tres ocupados pertenecen a los asalariados. Entre los asalariados, de nuevo se refleja el predominio del sector terciario en la economía urbana: $\mathbf{4 5 . 6} \%$ de los migrantes y casi la mitad $(48.8 \%)$ de la población nativa trabajan sólo como empleados en los sectores de comercio y servicios. Entre los trabajadores independientes, también la mayoría se desempeña en estos sectores, de tal manera que $20 \%$ de todos los migrantes y nativos ocupados $(20.4 \%$ entre los migrantes, $\mathbf{1 9 . 1} \%$ entre los nativos) se encuentran en este rubro.

CUADRO 4

Posición en el trabajo de los migrantes y de la población nativa en Tijuana, 1982

\begin{tabular}{lcc}
\hline Posición en el trabajo & Migrantes & $\begin{array}{c}\text { Población } \\
\text { nativa }\end{array}$ \\
\hline Profesionistas y técnicos & 9.5 & 13.0 \\
Empleados & 53.3 & 56.4 \\
$\quad$ Comercio & 22.8 & 22.1 \\
$\quad$ Servicios & 22.8 & 26.7 \\
Obreros y artesanos & 13.0 & 7.7 \\
$\quad$ Transformación & 3.4 & 4.6 \\
Construcción & 5.8 & 0.8 \\
Independientes & 24.2 & 22.9 \\
Comercio & 9.4 & 6.1 \\
Servicios & 11.0 & 13.0 \\
Total & 100.0 & 100.0 \\
& $(381)$ & $(131)$ \\
\hline
\end{tabular}

Fuente: Jorge Carrillo y Lothar Witte, "Migración y transformación social: migrantes me xicanos en la frontera norte de México y en los Estados Unidos", Tijuana, 1984, materiales no publicados

También en lo que se refiere a los ingresos obtenidos, resulta que en general la situación es muy parecida para ambos grupos. Tanto entre la población nativa como entre los migrantes, poco más de la tercera parte $(35.9 \%)$ recibe menos de un salario mínimo. ${ }^{22}$ En el otro extremo de la escala, los migrantes ganan con mayor frecuencia que los nativos ingresos que por lo menos equivalen a dos salarios mínimos $(24.7 \%$ entre los migrantes contra $20.6 \%$ entre los nativos). En este conjunto, es notorio que los migrantes obtengan ingresos relativamente altos sobre todo en el

22 En el momento del levantamiento de datos, el salario minimo era de 280 pesos al día. Para el cálculo se consideran 30 días por mes. 
sector servicios: en él, más de $20 \%$ de los empleados migrantes y más de la mitad de los trabajadores independientes (21.8 y $52.4 \%$ ) reciben por lo menos dos salarios mínimos (las cifras correspondientes para la población nativa son 5.7 y $29.4 \%$ ). En el caso de los empleados, un posible factor es, por una parte, que los puestos de mayor jerarquía en las empresas e instituciones públicas se cubren muchas veces con personal proveniente de otras ciudades y, por otra parte, que los migrantes se trasladan con mayor frecuencia al otro lado de la frontera para emplearse allí en el sector servicios. En el caso de los trabajadores independientes, la elevada proporción de ingresos muy altos entre los migrantes constituye sólo una cara de la medalla, dado que alrededor de la cuarta parte $(23.6 \%)$ gana menos de la mitad del salario mínimo. En eso se refleja la heterogeneidad de la actividad independiente en el sector servicios, que incluye tanto a la burguesía como a los grupos marginales.

A pesar de que, por lo general, la situación laboral de los migrantes es por lo menos equivalente a la de la población nativa, parece que los migrantes están ocupados en mayor proporción que los nativos en áreas de actividad de carácter estacional. Según el estudio llevado a cabo por el IIS-UABC en 1984, mientras que los migrantes representan $70 \%$ de todos los ocupados, entre los obreros de la construcción y los jornaleros ascienden a 90 y a $84.6 \%$, respectivamente. Además, constituyen casi $90 \%$ de los albañiles $(89.4 \%)$ y alrededor de $75 \%$ de los mozos, carpinteros y mecánicos, áreas en las cuales a menudo sólo se realiza una actividad temporal (desde $34 \%$ entre los albañiles hasta $58.9 \%$ entre los mecánicos). ${ }^{23}$

Esta situación laboral - que se puede resumir en que existe mayor heterogeneidad entre los migrantes que entre los nativos, tanto con respecto a los ingresos como a la seguridad en el empleo- probablemente refleja las diferencias entre las estructuras de edad y calificación de ambos grupos. Entre los migrantes, más de la mitad tienen por lo menos 30 años de edad. Casi $20 \%$ (19.3\%) no completó la primaria, otro $20 \%(19.7 \%)$ la completó pero no continuó sus estudios. En cambio, entre la población nativa, sólo el $13.5 \%$ tiene por lo menos 30 años de edad. ${ }^{24}$ Más de tres de cada cuatro nativos (77\%) por lo menos completó la secundaria, y en casi la mitad de los casos su formación condujo a una enseñanza especializada, orientada a oficios comerciales o técnicos, o a estudios universitarios (34.4 y 14.6\%, respectivamente). En una época en que el "credencialismo" y la búsqueda por una fuerza de trabajo joven se ha hecho todavía más fuerte en Tijuana, es lógico que la población nativa cumpla los criterios de reclutamiento en mayor medida que los migrantes.

${ }^{23}$ IIS-UABC (1985), cálculos propios.

${ }^{24}$ Esta cifra tan baja se explica por el hecho de que antes de la década de los cincuenta, cuando estas personas nacieron, vivían en Tijuana relativamente pocas personas en edad reproductiva. 
No obstante, es importante repetir que, a pesar de la mayor heterogeneidad entre los migrantes y a pesar de sus desventajas en términos de edad y calificación, en lo que se refiere a su posición en el mercado de trabajo y a los ingresos obtenidos, no son las diferencias con respecto a los nativos sino los rasgos comunes lo que constituye el resultado principal de la comparación de los datos.

Antecedentes socioeconómicos de los migrantes y su posición en el mercado de trabajo

Hasta aquí, uno de nuestros objetivos principales ha sido discutir la relación entre la migración y la estructura social. Como se mencionó, los migrantes hacia la ciudad de Tijuana provienen en gran parte de las regiones más desarrolladas del país; muchos hicieron sus experiencias laborales como asalariados antes de trasladarse hacia la frontera. En lo que sigue, se discutirá la relación entre estos antecedentes de los migrantes y su posición en el mercado de trabajo de Tijuana. Por un lado, se presentan los principales rasgos de la diferenciación entre los migrantes según sus regiones de procedencia. Por otro lado, se considera en qué medida sus experiencias laborales influyeron en su absorción en el mercado de trabajo.

Experiencias laborales anteriores y posición en el mercado de trabajo

Después de la segunda guerra mundial se pueden observar en México dos interesantes procesos sociales. En primer lugar, la transformación de la estructura del mercado de trabajo, donde el trabajo asalariado se expandió en términos absolutos y relativos. En segundo lugar, un proceso de urbanización intensamente ligado a la migración rural-urbana. ${ }^{25}$ En la medida en que el empleo en el campo está menos caracterizado por el trabajo asalariado que en la ciudad, ha de suponerse que la migración contribuye de manera considerable a la expansión del trabajo asalariado.

En el caso de la migración interna a Tijuana es interesante observar que, según los datos presentados en el cuadro 5 , no se puede afirmar que la migración contribuye a la expansión del trabajo asalariado en la economía mexicana. Entre los migrantes ocupados tanto en su región de procedencia como en Tijuana, el porcentaje de los asalariados disminuye con la migración. Antes de la migración, más de $70 \%$ se desempeñaban como empleados, obreros o jornaleros; mientras que, en Tijuana, menos de $60 \%$ vuelve a tener una ocupación parecida. Correspondientemente, el porcentaje de productores independientes (incluidos artesanos y pequeños cam-

\footnotetext{
25 Para un resumen de los rasgos generales de ambos procesos véase Klagsbrunn (1986b).
} 
pesinos) aumenta y de apenas un cuarto pasa a representar un tercio del total de los ocupados. ${ }^{26}$

CUADRO 5

Experiencias laborales anteriores y posición en el trabajo de los migrantes internos entrevistados en 1982 en Tijuana (I)

\begin{tabular}{lcc}
\hline Posición en ei trabajo & $\begin{array}{c}\text { Posición en el trabajo } \\
\text { en la región de } \\
\text { procedencia }\end{array}$ & $\begin{array}{c}\text { Posición en el } \\
\text { trabajo } \\
\text { en Tijuana, 1982 }\end{array}$ \\
\hline Empleados & 46.0 & 46.0 \\
Obreros & 10.9 & 11.8 \\
Jornaleros agrícolas & 14.7 & - \\
Pequeños propietarios & 3.8 & - \\
agrícolas & 2.8 & 3.8 \\
Artesanos & 15.6 & 30.3 \\
Independientes & 6.2 & 8.1 \\
Profesionistas y técnicos & 100.0 & 100.0 \\
Total & $(211)$ & $(211)$ \\
\end{tabular}

Fuente: Jorge Carrillo y Lothar Witte, "Migración y transformación social: migrantes mexicanos en la frontera norte de México y en los Estados Unidos", Tijuana, 1984, materiales no publicados.

Estos resultados agregados todavía permiten dos interpretaciones posibles. Por un lado podría ser que casi todo el grupo de los que anteriormente trabajaron como asalariados o como independientes vuelvan a trabajar en la misma posición también en Tijuana. Por otro lado, también podría ser que una parte considerable de los grupos respectivos pase de una posición a la otra.

En el análisis de las experiencias laborales anteriores de los grupos respectivos, presentado en el cuadro 6 se demuestra que la gran mayoría $(85 \%)$ de los migrantes que desempeñan una ocupación asalariada y que ya estaban ocupados en su región de procedencia, trabajaban también allí como asalariados. Sólo poco más de $10 \%$ eran antes independientes. Dentro de los asalariados, a su vez puede diferenciarse entre empleados y obre-

${ }^{26}$ No es posible una división de los ocupados en la agricultura en pequeños campesinos y jornaleros. En esta área trabajan sólo dos migrantes, lo que corresponde a menos de $1 \%$ del total de los migrantes ocupados. De acuerdo con la estructura probable que podrían haber desempeñado una tarea asalariada. En el cuadro no se les ha incluido por separado, sino que fueron sumados a los obreros. 
ros. De los migrantes que en Tijuana trabajan como empleados, $65 \%$ también lo habían hecho en su lugar de origen, mientras que $12.4 \%$ se desempeñaban como obreros y $10.3 \%$ como jornaleros. Entre los que son obreros en Tijuana, ni un solo individuo se había desempeñado antes como empleado; $32 \%$ habían sido antes también obreros y $44 \%$ jornaleros rurales (Carrillo y Witte, 1984). Correspondientemente, entre los independientes una parte considerable también era independiente antes de la migración.

\section{CUADRO 6}

Experiencias laborales anteriores y posición en el trabajo de los migrantes internos entrevistados en 1982 en Tijuana (II)

\begin{tabular}{|c|c|c|c|c|}
\hline \multirow[b]{2}{*}{$\begin{array}{c}\text { Posición en el trabajo } \\
\text { en Tijuana, } 1982\end{array}$} & \multicolumn{4}{|c|}{ Posición en ei trabajo en la región de procedencia } \\
\hline & $\begin{array}{c}\text { Empleados, } \\
\text { obreros y } \\
\text { jornaleros }\end{array}$ & $\begin{array}{l}\text { Pequeños propietarios } \\
\text { agrícolas, artesanos } \\
\text { e independientes }\end{array}$ & $\begin{array}{l}\text { Profesionistas } \\
\text { y técnicos }\end{array}$ & Total \\
\hline Empleados y obreros & 85.2 & 11.5 & 3.3 & $\begin{array}{l}100.0 \\
(122)\end{array}$ \\
\hline $\begin{array}{l}\text { Artesanos e } \\
\text { independientes }\end{array}$ & 54.2 & 43.1 & 2.8 & $\begin{array}{r}100.0 \\
(72)\end{array}$ \\
\hline Profesores y técnicos & 47.1 & 11.8 & 41.2 & $\begin{array}{r}100.0 \\
(17)\end{array}$ \\
\hline
\end{tabular}

Fuente: Jorge Carrillo y Lothar Witte, "Migración y transformación social: migrantes mexicanos en la frontera norte de México y en los Estados Unidos", Tijuana, 1984, materiales no publicados.

Si se considera esta relación desde la perspectiva de la región de procedencia, entonces la pregunta recibe otra formulación: ¿qué se hizo en Tijuana de los migrantes que antes habían sido independientes y asalariados? El cuadro 7 ilustra que dos de cada tres de los antiguos pequeños campesinos, artesanos y otros independientes ocupan en Tijuana posiciones semejantes, y sólo apenas $30 \%$ trabajan como empleados u obreros. En cambio, casi $70 \%$ de los anteriores empleados, obreros y jornaleros desempeñan en Tijuana una ocupación asalariada, y sólo un cuarto se dedica a un trabajo independiente.

Para resumir, en lo que respecta a la relación entre la estructura del mercado de trabajo y la migración, los principales resultados de este estu- 
CUADRO 7

Experiencias laborales anteriores y posición en el trabajo de los migrantes interne $s$ entrevistados en 1982 en Tijuana (III)

\begin{tabular}{lcccr}
\hline & \multicolumn{3}{c}{ Posición en ei trabajo en Tijuana, 1982 } \\
\cline { 2 - 5 } $\begin{array}{l}\text { Posición en el trabajo en } \\
\text { la región de procedencia }\end{array}$ & $\begin{array}{c}\text { Empleados } \\
\text { y obreros }\end{array}$ & $\begin{array}{c}\text { Artesanos e } \\
\text { independientes }\end{array}$ & $\begin{array}{c}\text { Profesionistas } \\
\text { y técnicos }\end{array}$ & Total \\
\hline $\begin{array}{l}\text { Empleados, obreros y } \\
\text { jornaleros agrícolas }\end{array}$ & 68.9 & 25.8 & 5.3 & 100.0 \\
$\begin{array}{l}\text { Pequeños propietarios } \\
\text { agrícolas, artesanos e }\end{array}$ & 29.8 & 66.0 & & $(151)$ \\
$\begin{array}{l}\text { indepéndientes } \\
\text { Profesionistas y técnicos }\end{array}$ & 30.8 & 15.4 & 53.8 & 100.0 \\
& & & & $(47)$ \\
\end{tabular}

Fuente: Jorge Carrillo y Lothar Witte, "Migración y transformación social: migrantes mexicanos en la frontera norte de México y en los Estados Unidos", Tijuana, 1984, materiales no publicados.

dio son los siguientes: en primer lugar, entre los migrantes ocupados tanto antes de la migración como en Tijuana, el predominio del trabajo asalariado era más importante en las regiones de procedencia que en Tijuana. En segundo lugar, en lo que se refiere a la transición del trabajo independiente al trabajo asalariado o viceversa, existe sólo una flexibilidad limitada. Entonces, sobre la base de nuestro ejemplo de la migración permanente a Tijuana, no se puede afirmar que la migración contribuya a la expansión del trabajo asalariado en la economía mexicana.

Procedencia regional y posición en el mercado de trabajo

En el diseño de este estudio se partió de la hipótesis de que el origen regional de los migrantes se refleja tanto en sus niveles de calificación como en sus experiencias laborales. La absorción de los migrantes en el mercado de trabajo se determina a su vez por las características socioeconómicas y las experiencias laborales anteriores de los migrantes. Por lo tanto, una comparación de la posición de los migrantes en el mercado de trabajo de Tijuana según su origen regional promete ofrecer otra perspectiva al conjunto.

Con base en los resultados presentados, no sorprende que los migrantes de regiones más desarrolladas se integren al mercado de trabajo en forma diferente que aquellos que provienen de regiones menos desarrolladas. En el cuadro 8 se puede observar, por ejemplo, que en el caso de los migrantes que anteriormente vivieron en la ciudad de México, casi $20 \%$ se desempeñan en Tijuana como profesionistas y técnicos, mientras que los 
valores correspondientes para migrantes del noroeste y del centro-oeste son un poco más del 11 y $4 \%$, respectivamente. La otra cara de la moneda se revela en el alto porcentaje de obreros y artesanos entre los que vinieron del centro-oeste, mientras que este porcentaje se encuentra más bajo en promedio para los migrantes de otras regiones. Las diferencias en la integración al mercado de trabajo se reflejan también en la estructura de los ingresos. Casi la mitad (46.6\%) de los migrantes del centro-oeste gana menos de un salario mínimo, mientras que esto se verifica sólo para alrededor de un cuarto (26.3\%) de los migrantes de la ciudad de México y para la tercera parte (32.4\%) de los del noroeste (Carrillo y Witte, 1984). En resumen, en lo que respecta tanto a la posición laboral como a los ingresos logrados en Tijuana, existen diferencias importantes entre los migrantes de las principales regiones de procedencia.

CUADRO 8

Posición en el trabajo de los migrantes internos procedentes de regiones seleccionadas en Tijuana, 1982

\begin{tabular}{lrrrr}
\hline & \multicolumn{3}{c}{ Región de procedencia } \\
\cline { 2 - 5 } \multicolumn{1}{c}{ Posición en el trabajo } & & & & Ciudad \\
\multicolumn{1}{c}{ en Tijuana } & $\begin{array}{r}\text { Total de } \\
\text { regiones }\end{array}$ & Noroeste & $\begin{array}{c}\text { Centro- } \\
\text { oeste }\end{array}$ & $\begin{array}{c}\text { México } \\
\text { Profesionistas y técnicos }\end{array}$ \\
Empleados & 9.4 & 11.3 & 4.2 & 19.3 \\
$\quad$ Comercio & 53.3 & 54.9 & 52.5 & 49.1 \\
$\quad$ Servicios & 22.8 & 25.4 & 24.6 & 14.0 \\
Obreros y artesanos & 22.8 & 22.5 & 19.5 & 29.3 \\
$\quad$ Industria de transformación & 13.1 & 10.6 & 17.0 & 7.1 \\
$\quad$ Construcción & 3.4 & 4.2 & 2.5 & 3.5 \\
Independientes & 5.8 & 3.5 & 9.3 & 1.8 \\
$\quad$ Comercio & 24.1 & 23.2 & 26.3 & 24.6 \\
Servicios & 9.4 & 5.6 & 13.6 & 17.6 \\
Total & 11.0 & 13.4 & 9.3 & 5.3 \\
& 100.0 & 100.0 & 100.0 & 100.0 \\
& $(381)$ & $(142)$ & $(118)$ & $(57)$. \\
\hline
\end{tabular}

Fuente: Jorge Carrillo y Lothar Witte, "Migración y transformación social: migrantes mexicanos en la frontera norte de México y en los Estados Unidos", Tijuana, 1984, materiales no publicados.

Es probable que estas diferencias estén estrechamente relacionadas con la calificación y las experiencias laborales anteriores de los migrantes de las respectivas regiones. En primer lugar, en lo que respecta a la califi- 
cación, en comparación con los migrantes de otras regiones, los del centrooeste sólo ofrecen cal ificaciones relativamente limitadas. Más de $30 \%$ carece de escuela primaria completa; otra cuarta parte de los migrantes provenientes de esta región la completó, pero no la secundaria. Sólo apenas un tercio de estos migrantes ha completado la secundaria o aprobado alguna formación posterior. Por el contrario, casi la mitad de los migrantes del noroeste y más de $70 \%$ de los de la ciudad de México terminaron al menos la secundaria (Carrillo y Witte, 1984). En segundo lugar, las experiencias laborales de los migrantes en sus regiones de procedencia eran diversas también. Según los datos presentados en el cuadro 9, un tercio de los migrantes del centro-oeste trabajaban en la agricultura, en su mayoría como jornaleros (22.7\%), y la sexta parte como obreros o artesanos. En cambio, sólo un tercio logró la posición de empleado. Por el contrario, más de la mitad de los migrantes procedentes del noroeste habían sido empleados, y entre los migrantes de la ciudad de México alrededor de $60 \%$ habian trabajado como empleados y otro $15 \%$ como profesionistas y técnicos.

CUADRO 9

Experiencias laborales anteriores de los migrantes internos entrevistados en 1982, en Tijuana procedentes de regiones seleccionadas

\begin{tabular}{lrrrr}
\hline & \multicolumn{3}{c}{ Región de procedencia } & \\
\cline { 2 - 5 } Posición en el trabajo en & $\begin{array}{c}\text { Total de } \\
\text { las re- } \\
\text { giones }\end{array}$ & Noroeste & Centro-oeste & $\begin{array}{c}\text { Ciudad } \\
\text { de } \\
\text { la región de procedencia }\end{array}$ \\
\hline Profesionistas y técnicos & 5.4 & 4.6 & - & 15.5 \\
Empleados & 45.4 & 52.3 & 34.6 & 578 \\
$\quad$ Comercio & 16.5 & 21.6 & 12.0 & 22.2 \\
$\quad$ Servicios & 17.7 & 19.3 & 12.0 & 22.2 \\
Obreros y artesanos & 13.8 & 79 & 17.3 & 6.7 \\
$\quad$ Ind. de transformación & 5.0 & - & 4.0 & 6.7 \\
$\quad$ Construcción & 5.0 & 4.5 & 8.0 & - \\
Ocupados en agricultura & 17.7 & 15.9 & 33.4 & 2.2 \\
$\quad$ Jornaleros & 13.1 & 12.5 & 22.7 & 2.2 \\
Independientes & 17.7 & 19.3 & 14.7 & 17.7 \\
$\quad$ Comercio & 3.1 & 5.7 & 2.7 & 2.2 \\
$\quad$ Servicios & 8.8 & 10.2 & 6.7 & 4.4 \\
Total & 100.0 & 100.0 & 100.0 & 100.0 \\
& $(260)$ & $(88)$ & {$[75]$} & $(45)$ \\
\hline
\end{tabular}

Fuente: Jorge Carrillo y Lothar Witte, "Migración y transformación social: migrantes mexicanos en la frontera norte de México y en los Estados Unidos" , Tijuana, 1984, materiales no publicados. 
Se confirma así que para migrantes de regiones menos desarrolladas como el centro-oeste, tanto por su limitada calificación formal como por sus experiencias laborales anteriores, existe una menor posibilidad que para migrantes de la ciudad de México o del noroeste del pais, de integrarse en el mercado de trabajo de Tijuana de una manera que les ofrezca las mismas oportunidades de asegurar su reproducción.

\section{Consideraciones finales}

En este trabajo se ha sostenido que Tijuana, una de las principales ciudades mexicanas, ha constituido el prototipo de un área urbana que crece por migración interna. En los años ochenta, alrededor de dos de cada tres residentes eran migrantes.

Los migrantes vienen principalmente de regiones relativamente desarrolladas: los del noroeste, de la ciudad de México y del centro-oeste representan la gran mayoría de todos los migrantes, y más de la cuarta parte viene sólo de las dos metrópolis de México y Guadalajara.

Las experiencias laborales de los migrantes en sus regiones de procedencia corresponden al carácter relativamente desarrollado de esas regiones. Casi dos de cada tres migrantes entre los que trabajaron antes de la migración, lo hicieron en posiciones asalariadas, desempeñándose $40 \%$ como empleados. En lo que se refiere a las diferencias regionales, resulta que las experiencias laborales individuales reflejan el carácter de las respectivas regiones de procedencia. Entre los migrantes de las regiones más desarrolladas, el trabajo asalariado y especialmente el trabajo en calidad de empleado es más pronunciado que en otras regiones.

En el mercado de trabajo de Tijuana, los migrantes, por lo general, trabajan en los mismos sectores y en las mismas posiciones que la población nativa. Correspondiendo a la estructura de la economía urbana a principios de los años ochenta, tal panorama está dominado por el sector terciario, en el cual trabajan alrededor de dos de cada tres ocupados. El trabajo asalariado predomina en todas las ramas de la economía con alrededor de 70 por ciento.

Entre los migrantes que trabajan tanto en sus regiones de procedencia como en Tijuana, sorprende que en esta ciudad tengan una ocupación asalariada con menor frecuencia que antes de la migración.

En lo que se refiere a las diferencias en relación a la procedencia regional de los migrantes, destaca que entre los migrantes de regiones más desarrolladas el porcentaje de los que trabajan en Tijuana, sea como asalariados - sobre todo empleados - sea como profesionistas y técnicos, es más alto que entre los migrantes de otras regiones. Este resultado refleja tanto las diferencias en las experiencias laborales anteriores como en los niveles de calificación formal. 
En vista de estos resultados, podemos plantear para el caso de la migración interna a Tijuana, según los conceptos y fuentes empleados en este estudio, que el tipo de migración tiende a parecerse al que tiene lugar en los países industrializados más que en los países en vías de desarrollo. Por una parte, predomina el movimiento entre regiones urbanizadas y no el movimiento de las zonas rurales hacia la ciudad. Por otra son los asalariados $-\mathrm{y}$ entre ellos, sobre todo los empleados- y no los productores y comerciantes independientes, los que constituyen la gran mayoría de los migrantes. Por ende, en este caso la migración interna no contribuye a la expansión del trabajo asalariado.

Para evitar correr el peligro de una generalización prematura, cabría destacar que los resultados expuestos están considerablemente determinados por una situación específica en el mercado de trabajo, el cual, en las últimas décadas, se encontraba en condiciones de expansión y diversificación considerables. Sobre la base de un ritmo de crecimiento autónomo relativamente estable, en este proceso se han presentado múltiples posibilidades de trabajo para los migrantes.

No puede pronosticarse en qué medida Tijuana seguirá siendo en el futuro una ciudad abierta, para muchos relacionada con la esperanza, todavía justificada, de una vida mejor. Como fue expuesto en este trabajo, esto dependerá, en primera instancia, de la configuración histórica, del desarrollo del país y de la región, la cual, tal vez, otra vez dará sentido a este flujo migratorio.

\section{Apéndice}

Los materiales incluidos en el trabajo de Jorge Carrillo y Lothar Witte, “Migración y transformación social: migrantes mexicanos en la frontera norte de México y en los Estados Unidos", se basan en una investigación empírica realizada en los meses de marzo y abril de 1982 por el Centro de Estudios Fronterizos del Norte de México (Cefnomex), en seis ciudades de la región fronteriza del norte de México (Tijuana, Mexicali, Ensenada, Tecate, Ciudad Juárez, Nuevo Laredo). En total se encuestaron 2320 individuos con residencia permanente en estas ciudades. Como unidad de investigación se eligió al jefe de familia. A causa de la elección de esa unidad de investigación se da un predominio de la población masculina entre los encuestados.

A continuación se aclaran algunos conceptos empleados en ese trabajo:

Población nativa y migrante: se considera como población nativa a las personas que respondieron a la pregunta del lugar de nacimiento y de su última residencia identificándolos con el lugar en que fueron encuestados. Las demás personas se designaron como migrantes; también se consideraron como migrantes aquellos que nacieron en los respectivos luga- 
res de la región de la frontera norte y que, tras una migración temporal, regresaron a ellos. Este grupo representa, no obstante, sólo un pequeño porcentaje de la totalidad de los migrantes.

Región de procedencia: como región de procedencia de los migrantes se designa siempre la región en" que tuvieron su última residencia habitual antes de su migración a las ciudades de la región fronteriza. En todos los casos se reunieron varios estados en una región.

Calificación: los datos sobre la calificación se basan en los datos sobre la aprobación de los años escolares o de perfeccionamiento. La aprobación de un número determinado de años escolares corresponde a la aprobación de determinados ciclos de enseñanza. En particular, los conceptos se emplean de la siguiente manera: primaria no completa (menos de seis años de asistencia escolar); primaria completa (seis años); secundaria no completa (más de seis y menos de nueve años de asistencia escolar); secundaria completa (nueve años); enseñanza especializada (más de nueve años, sin asistencia a la universidad); formación universitaria (por lo menos un año completo en la Universidad).

Experiencias laborales anteriores/posición en el trabajo: en la categoría "Experiencias laborales anteriores", se encuentran aquellos migrantes que habían tenido una actividad económica en su región de procedencia. En lo que respecta a la agrupación "Posición en el trabajo", se toma en cuenta también la ocupación desempeñada en los municipios de la región fronteriza o en la región de procedencia. En primer lugar se trata de una división según profesionistas y técnicos, empleados, obreros, artesanos, trabajadores agrícolas, trabajadores por cuenta propia. Los grupos así constituidos originariamente incluidos en el cuestionario fueron parcialmente reunidos en grupos mayores. Se consideró adecuado un tratamiento conjunto de los llamados profesionistas y técnicos, que también fueron considerados conjuntamente en los censos mexicanos de población hasta el año 1970. Reflexiones similares llevaron a la fusión de obreros y artesanos. Tanto en 1960 como en 1980 ambos grupos fueron presentados conjuntamente en los censos. Además, los artesanos representan sólo un muy reducido porcentaje de la totalidad de los ocupados, de modo que los datos para el grupo de obreros y artesanos, incluso a pesar de que en el caso de los artesanos se trata sobre todo de independientes y rara vez de asalariados en el sector secundario, pudieron tomarse, generalizando, como asalariados. Los ocupados en la agricultura fueron luego subdivididos, diferenciando entre pequeños propietarios, ejidatarios o jornaleros. En este sentido pareció menos adecuada una división de los ocupados en la agricultura según la categoría de asalariados e independientes, ya que a menudo se ocupan posiciones tanto independientes como asalariadas. Como independientes se consideran todos aquellos ocupados que no pudieron clasificar su actividad en ninguno de los grupos mencionados, esto es, que no se desempeñaban ni como obreros ni como empleados, así como 
tampoco en la agricultura o como profesionistas o técnicos. Una comparación del porcentaje de los independientes así caracterizados con los resultados del censo de 1980 y también de otras investigaciones empíricas de la década de los ochenta permite comprobar la corrección de este procedimiento.

\section{Bibliografía}

Balán, J., H.L. Browning y E. Jelin (comps.) (1973), Migración, estructuro ocupacional y movilidad social (el caso de Monterrey), México, D. F.

Bustamante, J. (1982), Actitudes políticas de los fronterizos, Informe de investigación inédito, Tijuana.

(1984), "Migración interna e internacional y distribución del ingreso", en Comercio exterior, vol. 34, núm. 9, pp. 849-863.

Carrillo, J. (1984), La frontera norte y el movimiento obrero en la industria maquiladora, Tijuana, manuscrito inédito.

y L. Witte (1984), Migración y transformación social: migrantes mexicanos en la frontera norte de México y en los Estados Unidos, Tijuana/Berlín Occidental, materiales de proyecto inéditos.

Consejo Nacional de Población (1984), Estudio sociodemogrófico del Estado de Baja California, México.

Corona, R. (1983), Evaluación de los datos del Censo de 1980 sobre población residente, inmigración y emigración de Baja California, Tijuana.

Garibay, R. (1981), De lujo y hambre, México.

Guevara Santillán, M. (1979), Estadisticas y gráficas de Tijuana, Tijuana.

Harris, J. R. y M.P., Todaro (1970), "Migration, Unemployment and Development: A Two-Sector Analysis", en American Economic Review, vol. LX, pp. 126-142.

Henning, C. y U. Paulsdorff (1985), Indianische Kultur und ihre Adaption an das stadtische Unfeld: Die soziale Organisation der Mixteken, Colonia Obrera, Tijuana, Baja Galifornia, Mexiko, Tijuana, manuscrito inédito.

Hernández Laos, E. (1984), "La desigualdad regional en México (1900-1980)", en Cordera, R. y C. Tello (comps.), La desigualdad en México, México, pp. 155-192.

Instituto de Investigaciones Sociales de la Universidad Autónoma de Baja California (1984), El trabajador mexicano en Estados Unidos: transmigración y desarrollo económico en la frontera Tijuana-San Diego, Mexicali, informe de investigación inédito.

(1985), Desarrollo urbano y fuerza de trabajo en la frontera norte de México: el caso de Tijuana, Baja California, Mexicali, informe de investigación inédito.

Klagsbrunn, V. (comp.) (1986a), "Lehrforschungsprojekt Mexiko: Veränderungen der Sozialstruktur und Migration in Mexiko", en Arbeitspapiere zu Wirtschaft, Gesellschaft und Politik in Entwicklungslandern, Nr. 5, Schwerpunktbereich Entwicklungssoziologie am Institut fur Soziologie der Freien Universitat Berlin, Berlín Occidental.

(1986), "Veränderung der Sozialstruktur und Migration in Mexiko: Eine erste Auswertung des Bevolkerungszensus von 1980", en Klagsbrunn, V. (comp.), "Lehrforschungsprojekt Mexiko: Veranderungen der Sozialstruktur und Mi- 
gration in Mexiko", en Arbeitspapiere zu Wirtschaft, Gasellschaft und Politik in Entwicklungslandern, Nr. 5, Schwerpunkbereich Entwicklungssoziologie am Institut fur Soziologie pp. 1-41 der Freien Universitat Berlin, Berlin Occidental.

Lewis, W.A. (1985), “Economic Development with Unlimited Supplies of Labour”, en The Manchester School of Economic and Social Studies,. vol. XXII, núm. 2, pp. 139-191.

Lipton, M. (1982), 'Migration from Rural Areas of Poor Countries: The Impact on Rural Productivity and Income Distribution", en R.H. Sabot (comp.), Migration and the Labor Market in Developing Countries, Boulder/Co., pp. 65-89.

Marx, K. (1979), Das Kapital. Kritik der politischen ökonomie, Erster Band, en Karl Marx y Friedrich Engels, Werke, Bd. 23, Berlín (RDA).

(1971), "Entwurf einer nicht gehaltenen Rede zur irischen Frage", en Karl

Marx y Friedrich Engels, Werke, Bd. 16, Berlin (RDA), pp. 439-458.

(1969), "Erzwungene Emigration-Kossuth und Mazzini-Die

Fluchtlingsfrage-Wahlbestechung in England-Mr. Cobden", en Karl Marx y Friedrich Engels, Werke, Bd. 8, Berlin (RDA), pp. 541-547.

Melesio Nolasco, J.C. (1983), “Migración y fuerza de trabajo en la frontera norte y Tijuana. Un estudio demográfico de la frontera norte de México, 1921-1980", México, tesis de licenciatura inédita.

Mungaray, A. y P. Moctezuma (1984), “'La disputa del mercado fronterizo 1960 1983", en Estudios fronterizos, año 1, núm. 3, Mexicali, pp. 89-111.

(1985), Distribución del ingreso, comportamiento del consumo y precios en el área urbana de Tijuana, B.C., Tijuana, informe de investigación inédito.

Muñoz, H., O. de Ohveira y C. Stern (comps.) (1977), Migración y desigualdad social en la ciudad de México, México.

Nacional Financiera (1984), La economía mexicana en cifras, México.

Price, J. (1973), "Tijuana: Urbanization in a Border Culture", Notre Dame/Ind.

Sabot, R.H. (1982), “Conclusion: Some Themes and Unresolved Issues", en R.H. Sabot (comp.), Migration and the Labor Market in Developing Countries, Boulder/Co., pp. 229-241.

Secretaria de Industria y Comercio (1976), Indicadores socioeconómicos de las zonas fronterizas, México.

Secretaría de Programación y Presupuesto (1983a), X Censo general de población y vivienda, 1980. Estado de Baja California, vol. 1, tomo II, México. (1983b), Estadística de la industria maquiladora de exportación, 1974-1982, México.

(1984), Estadísticas de la industria maquiladora de exportación, México.

Singer, P. (1973), “Migraciones internas en América Latina: consideraciones teóricas sobre su estudio", en M. Castells (comp.), Imperialismo y urbanización en América Latina, Barcelona, pp. 27-54.

Stark, O. (1982), "'Research on Rural-to-urban Migration in LDCs: The Confusion Frontier and why We Should Pause to Rethink Afresh", en World development, vol. 10, núm. 1, Londres, pp. 63-70.

Stern, C. (1977), "Migración, educación y marginalidad", en H. Muñoz, O. de Olivena y C. Stern (comps.), Migración y desigualdad social en la ciudad de México, México, pp. 101-112.

Todaro, M.P. (1969), “A Model of Labor Migration and Urban Unemployment in Less Developed Countries", en American Economic Review, vol. 59, pp. 138-148.

Unikel, L. (1978), El desarrollo urbano de México. Diagnóstico e implicaciones futuras, México, 2da. edición.

Witte, L. (1986a), “Tijuana, Baja California Norte. Entwicklung und Migration in 
einer Stadt der nördlichen mexikanischen Grenzregion", Berlin Occidental, tesis de maestría inédita.

(1986a), "Ein Migrant kommt selten allein. Zur Bedeutung sozialer Zusammenhange im MigrationsproseB", en V. Klagsbrunn (comp.), “Lehrforschungsproject Mexiko: Veranderungen der Sozialstruktur und Migration in Mexiko", en Arbeitspapiere zu Wirtschaft, Gesellschaft und Politik in Entwicklungslandern, Nr. 5, Schwerpunktbereich Entwicklungssoziologie am Institut fur Soziologie der Freien Universitat Berlin, Berlin Occidental, pp. 441-480.

Zazueta, César (1980), Investigación reciente sobre migración mexicana indocumentada a los Estados Unidos (la encuesta nacional de emigración a la frontera norte del país y a los Estados Unidos, ENEFNEU), Cambridge, Mass., manuscrito inédito. 


$$
\text { . }
$$

